\title{
Régulation en cis et en trans de la différenciation cellulaire
}

Il est maintenant parfaitement clair que l'activité des gènes est contrôlée par des protéines se fixant sur des motifs d'ADN particuliers des régions régulatrices (voir "la riche union de l'ADN et des protéines ", $m / s n^{\circ} 7$, vol. 3 , p. 428, et " $A D N$, protéines et transcription", $m / s n^{\circ} 8$, vol. 3, p. 487). En première analyse, cependant, cette constatation ne fait que repousser le problème de la différenciation puisque l'on peut se demander ce que sont les mécanismes contrôlant la synthèse et l'activité des protéines actives sur la transcription au cours du développement et de la différenciation. Par ailleurs, la diversité des programmes d'expression des gènes au cours du développement est extraordinaire : non seulement il existe un très grand nombre de types de différenciation cellulaire, mais encore ces types peuvent être divisés en de multiples périodes (in vivo, par exemple, périodes embryonnaire précoce, fotale, néonatale, post-natale, adulte, etc.) caractérisées par l'expression de gènes marqueurs spécifiques. De plus, au cours d'une même " période " du développement d'un tissu donné, l'expression de ces marqueurs spécifiques est souvent asynchrone. La question des mécanismes contrôlant cette impressionnante diversité de comportement des gènes, se pose donc avec acuité.

Au début de tout, la membrane. La membrane plasmique est la première cible des signaux " différenciateurs " aussi bien que des signaux mitotiques $\left(\mathrm{m} / \mathrm{s} n^{\circ} 5\right.$, vol. $3, p .174$ ) ; c'est elle qui, précocément dans l'embryogenèse, va " percevoir " un gradient de morphogènes $(m / s \quad n \circ 5$, vol. 3 , p. 178), va lier sur un récepteur spécifique un facteur de différen- ciation $\left(\mathrm{m} / \mathrm{s} n^{\circ} 6\right.$, vol. 2, p. 340), ou bien va recevoir une information par contact direct avec les cellules adjacentes.

On savait déjà que certains gènes intervenant dans le développement embryonnaire codent pour des molécules étrangement proches des facteurs de croissance (produits des gènes notch et lin $12, \mathrm{~m} / \mathrm{s} n^{\circ} 6$, vol. 2, p. 340), d'autres pour des protéines ressemblant aux protéoglycanes de la matrice extracellulaire (gène per, voir mini-synthèse sur les canaux de jonction intercellulaire dans ce numéro). Plus récemment, l'équipe de G.M. Rubin en Californie a pu déterminer la structure du gène sevenless de la drosophile, impliqué dans la différenciation des photorécepteurs. $\mathrm{Ce}$ gène a le potentiel de coder pour un récepteur membranaire doté d'une activité tyrosine-kinase [1], comme tant de récepteurs de facteurs de croissance et de produits d'oncogène $\left(m / s n^{\circ} 8\right.$, vol. $3, p$. 492). Les gènes du développement et les oncogènes ne sont d'ailleurs pas de nature différente et tout indique que certains oncogènes sont probablement impliqués dans la différenciation cellulaire $\left(m / s n^{\circ} 4\right.$, vol. 2 , p. 222).

Modifications post-traductionnelles des facteurs transcriptionnels. Il y a peu encore, ce qui se passait entre la perception par une cellule d'un signal membranaire et la réponse transcriptionnelle au niveau du noyau, constituait un véritable " trou noir". Le mode d'action des hormones stéroïdes suggérait cependant un modèle simple de modulation de l'activité d'un gène par activation, sous l'effet d'un inducteur externe (l'hormone), d'un facteur transcriptionnel pré-existant (le récepteur de l'hormone, $m / s \quad n^{\circ} 3$, vol. 3, p. 172). Dans ce cas, cependant, l'hormone pénètre dans la cellule sans intervention d'un récepteur membranaire et de " seconds messagers". Chez E. coli, la réponse transcriptionnelle à des stimuli extérieurs (modification du milieu de culture), comporte la synthèse d'AMP cyclique qui se fixe à un facteur transcriptionnel pré-existant (la protéine CAP) et l'active [2]. De la même manière, l'activation du répresseur de l'opéron tryptophane est due à une modification de la structure de la protéine, provoquée par la liaison d'une molécule de tryptophane [3] ; la trans-conformation ainsi induite rend le répresseur apte à reconnaître le site opérateur qui contrôle la transcription de l'opéron. Depuis quelques mois, il est apparu qu'un tel modèle de l'activation, par un inducteur, d'un facteur transcriptionnel pré-existant était probablement de portée générale. Le enhancer du gène codant pour la chaîne légère kappa des immunoglobulines (IgG-x) n'est actif que dans les cellules lymphocytaires $B$; son activation est corrélée avec la formation d'un complexe entre un motif du enhancer (motif $\mathrm{B}$ ) et la protéine $\mathrm{NF}_{-} \mathrm{B}$. Cette protéine, en fait, n'est absolument pas spécifique des lymphocytes B et peut être détectée dans toutes les cellules où elle est cependant incapable de se fixer au motif B du enhancer IgG- $x$. Le traitement des cellules non différenciées dans la voie des lymphocytes B par des activateurs de la protéine kinase $\mathrm{C}\left(\mathrm{m} / \mathrm{s} n^{\circ} 3\right.$, vol. 3, p. 174) active NF- ${ }_{x} \mathrm{~B}$, c'est-à-dire la rend capable de se fixer à son site d'ADN [4]. Deux autres facteurs transcriptionnels, probablement impliqués dans la réponse transcriptionnelle de certains gènes lors de la stimulation de la prolifération cellulaire, pour- 
raient aussi être activés par la modification de molécules préexistantes inactives : les protéines SRF, se fixant notamment sur le enhancer de l'oncogène $c$-fos $[5,6]$ et AP1 [7, 8], dont plusieurs cibles sont connues. Dans ces exemples, cependant, " l'activation " des facteurs transcriptionnels ne semble pas, au moins in vitro, agir sur leur capacité de liaison à l'ADN, mais plutồt sur leur aptitude à interagir avec le complexe d'initiation de la transcription.

Les facteurs transcriptionnels probablement responsables de la réponse des gènes métallothionéine aux métaux lourds (MRE-binding protein, protéine se liant à l'élément de réponse aux métaux) [9] et de la réponse à la chaleur des gènes codant pour les protéines du choc thermique (HSF, hedt shock transcriptional factor) [10] sont également activés après leur synthèse (activation post-traductionnelle).

Un nombre peut-être limité de facteurs... Les travaux qui se multiplient sur la mise en évidence et la caractérisation de protéines pouvant jouer un rôle dans le contrôle de l'expression des gènes, débouchent sur deux constatations à première vue paradoxales : les mêmes facteurs sont souvent retrouvés impliqués dans le contrôle de gènes fort différents, et certains facteurs quoiqu'ils reconnaissent un motif indispensable à l'expression d'un gène spécifique d'un type particulier de différenciation, sont en fait parfaitement ubiquitaires (voir l'exemple de $\mathrm{NF}-\mathrm{B}$ ). C'est ainsi que la protéine IgNF-A, qui reconnaît la séquence octamérique dont nous avons parlé dans la première nouvelle de cette série $\left(m / s \quad n^{\circ} 7\right.$, vol. 3, p. 428) joue un rôle essentiel dans l'activation du enhancer des chaînes lourdes des immunoglobulines au cours de la différenciation des lymphocytes $\mathrm{B}$, mais aussi dans le fonctionnement d'autres promoteurs [12]. La répartition d'IgNF-A est, de plus, ubiquitaire*. Deux autres exemples illustrent ce phénomène de $m / s n^{\circ} 9$ ool. 3, noocmbre 87 l'intervention possible de mêmes facteurs dans des types différents de régulation; ils concernent les facteurs SRF et AP1, dont nous avons parlé plus haut. SRF se fixe sur le enhancer de l'oncogène $c$-fos et en active probablement ainsi la transcription $[5,6]$. Françoise Phan Dinh Tuy et Adrian Minty, à Paris, ont montré que ce même facteur se liait à des séquences situées en amont des gènes d'actine musculaire et indispensables à l'activation de la transcription de ces gènes au cours de la différenciation musculaire (communication personnelle). Une protéine impliquée dans la réponse de gènes à l'AMP cyclique a été récemment caractérisée [13] ; elle reconnaît une séquence pratiquement identique à celle reconnue par AP1 [7, 8] et, de plus, lorsqu'un gène est contrôlé à la fois par les esters de phorbol** et l'AMP cyclique, cette même séquence est indispensable aux deux types de contrôle [4]. Ces résultats suggèrent donc que la protéine AP1 et la protéine responsable de la réponse à l'AMP cyclique pourraient être identiques.

Création de diversités. La diversité des contrôles transcriptionnels auxquels sont soumis les gènes peut naturellement être le reflet de la diversité des facteurs régulateurs. Il faut remarquer qu'une telle solution serait peu "économique " puisqu'il faudrait alors envisager, en poussant cette hypothèse jusqu'à l'absurde, l'existence d'un gène régulateur par gène effecteur, ainsi que des gènes contrôlant les gènes régulateurs! Nous avons, de plus, déjà signalé que les mêmes facteurs intervenaient souvent dans le contrôle de gènes différents.

Dans certains cas, cependant, des facteurs transcriptionnels semblent bien "spécifiques " de tissus don-

\footnotetext{
- Il existerait, en fait, deux espèces d'IgNF-A, l'une ubiquitaire et l'autre caractéristique des cellules $B$. * Les esters de phorbol sont des "promoteurs "de la cancérogenèse. Ils agissent en activant la protéine kinase $C$.
}

nés... ce qui revient à dire qu'ils y sont en concentration nettement plus élevée qu'ailleurs [15-17]. $\mathrm{La}$ combinaison variable $\mathrm{d}^{3} \mathrm{un}$ petit nombre de facteurs régulateurs peut, elle aussi, engendrer une considérable diversité... celle des mots innombrables composés à l'aide des lettres d'un alphabet $[11,16,17]$. De fait, "la dissection fonctionnelle " des enhancers, c'est-à-dire l'étude des effets de mutations ou de microdélétions en différents sites, montre qu'ils sont constitués de cassettes, chacune interagissant probablement avec un facteur protéique et ayant individuellement une spécificité qui peut être différente de celle du enhancer total [18-20]. Le enhancer du virus SV40 contrôlant l'expression du gène $\mathrm{T}$ est ainsi constitué : de l'octamère liant le facteur IgNF-A et conférant à des gènes en amont desquels il est inséré en plusieurs copies, une spécificité lymphoïde d'expression; de deux autres éléments qui, analysés de la même manière que l'octamère, confèrent soit, pour l'un, une spécificité pour des cellules de rein de singe, soit, pour l'autre, une expression ubiquitaire [20].

Un même facteur peut être variablement modifié (par phosphorylation, par exemple) et interagir de façon différente selon le type de modification subie. Ainsi, avons-nous vu que des facteurs inactifs pouvaient être activés. Dans le cas d'AP1, s'il s'avérait que cette protéine était également impliquée dans la régulation par l'AMP cyclique, on pourrait imaginer qu'elle a des effets différents et (ou) interagit avec des motifs différents selon qu'elle est phosphorylée à un site sous l'action de la protéine kinase C ou à un autre site sous l'action d'une protéine kinase stimulée par l'AMP cyclique.

Enfin, dernier élément engendrant de la diversité, un même facteur peut reconnaître des motifs d'ADN voisins, mais non identiques, et contracter des interactions légèrement différentes les unes des autres selon le motif de 
liaison. Dans ce cas, le facteur " actif en trans" est le même pour plusieurs gènes et la diversité de réponse fonctionnelle est due aux différences de séquence des motifs reconnus; il s'agit donc ici d'un contrôle en cis de la spécificité d'expression. La fixation variable de SRF en amont du gène $c$-fos ou du gène actine musculaire pourrait peut-être constituer un exemple de ce mécanisme (Francoise Phan Dunh Tuy et Adrian Minty, communication personnelle) : dans le premier cas, cette fixation confererait la réponse à l'induction de la prolifération alors que, dans le second cas, elle interviendrait dans l'expression au cours de la différenciation musculaire.

Un schéma unifié. La figure 1 présente une vue unifiée de la réponse transcriptionnelle à l'induction de la différenciation... ou de la prolifération cellulaire. Un signal perçu par la membrane (étape 1) entraîne, via la synthèse de seconds messagers, la modification post-traductionnelle d'un facteur transcriptionnel préexistant (étape 2). Cette activation $\left(\mathrm{F}^{\mathrm{A}} \rightarrow \mathrm{F}_{\mathrm{a}}^{\mathrm{A}}\right.$ dans la figure) modifie la transcription de plusieurs gènes,

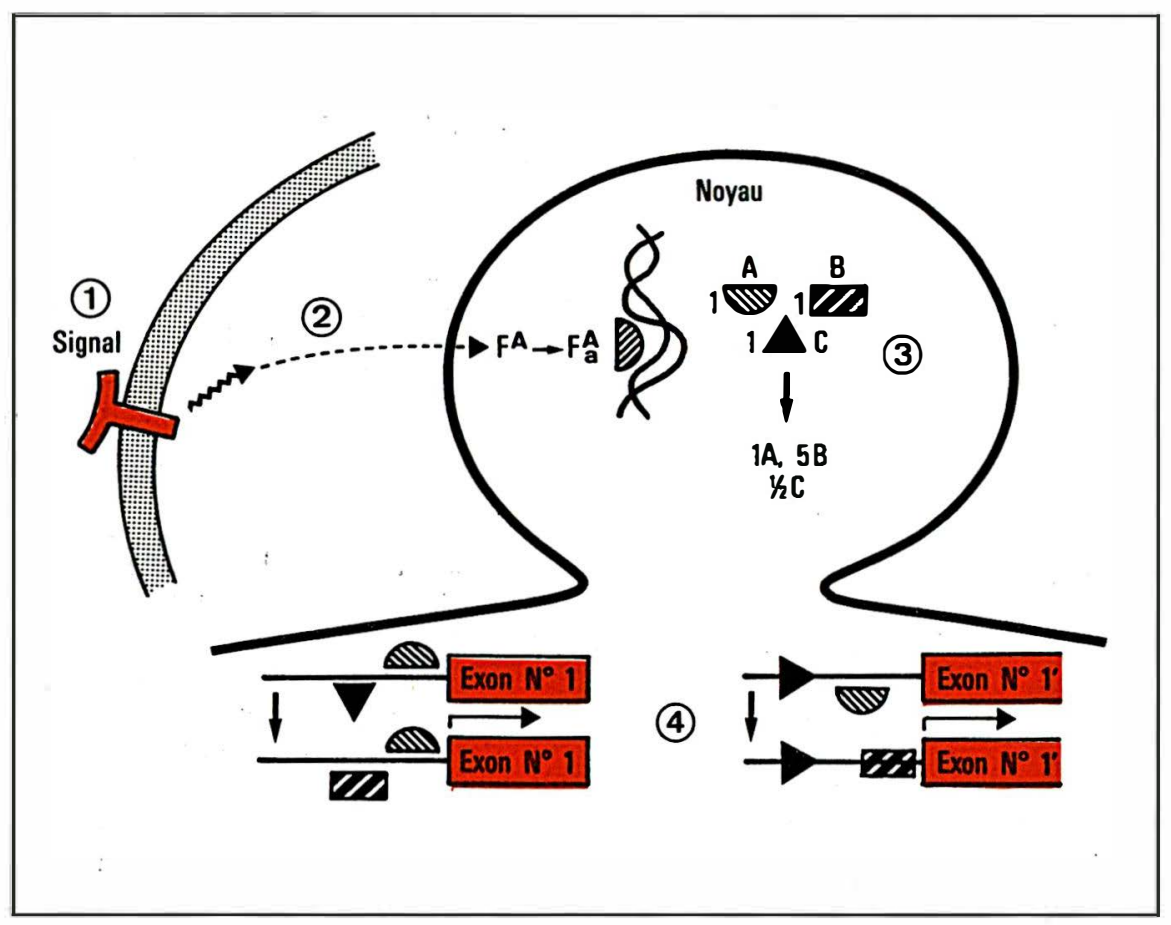

Figure 1. Réponses transcriptionnelles en cascade à un inducteur de différenciation ou de prolifération. Pour l'explication détaillée du schéma, voir texte, dernier paragraphe. $A, B, C$ : facteurs transcriptionnels; $F_{a}^{A}$ : facteur $A$ actif, se fixant sur la double hélice $d^{\prime} A D N$ et entraînant un effet transcriptionnel ; étape 3 : changement quantitatif des facteurs $A, B$ et $C$ qui, initialement en proportion équimolaire, se retrouvent dans la proportion $1 A, 5 B$ et $1 / 2 C$, après la réponse transcriptionnelle primaire due à l'activation $F^{A} \rightarrow F_{g}^{A}$; létape 4 représente la réponse transcriptionnelle secondaire à la modification quantitative des facteurs $A, B$ et $C$; à gauche, le site de reconnaissance de $B$ chevauche celui de $C$, si bien que l'augmentation du rapport B/C aboutit à un déplacement de $C$. A droite, $B$ déplace $A$ et est combiné avec les autres facteurs de façon différente de celle représentée à gauche. De plus, le facteur $C$ peut avoir des interactions variées avec des séquences voisines mais différentes. La combinaison de tous ces phénomènes crée une grande diver-

dont certains codent eux-mêmes pour des facteurs transcriptionnels A, B et C. De ce fait, la proportion relative de ces facteurs se modifie (étape 3), ce qui aboutit (étape 4) à la modification des combinaisons de facteurs liés en amont des gènes qui sont, de ce fait, activés ou inactivés. Ce schéma, sûrement beaucoup trop simple (et rappelons ici que certains facteurs " spécifiques de tissus " ont été décrits), suppose l'existence de phénomènes de régulation en cascade dont un exemple pourrait être la voie à laquelle appartient $c$-fos : un phénomène d'activation post-traductionnelle de SRF explique peut-être l'augmentation de la synthèse de $c$-fos qui est, elle-même, une protéine se liant à l'ADN et pouvant contrôler l'expression d'une deuxième classe de gènes [21]. Ce schéma $a$, en revanche, l'interêt de concilier l'essentiel des données récentes acquises en ce domaine : les premiers événements de l'induction de la différenciation sont membranaires ; la réponse transcriptionnelle précoce de certains gènes ne nécessite pas la synthèse de nouvelles protéines; les mêmes facteurs variablement combinés sont souvent trouvés associés au contrôle de l'expression de gènes impliqués dans des types très différents de différenciation; enfin, certaines des protéines codées par les gènes précocément activés au cours de ces phénomènes sont elles-mêmes des facteurs transcriptionnels jouant un rôle probable dans l'activité d'autres gènes [22].

Axel Kahn

\section{RÉFÉRENCES}

1. Hafen E, Basler K, Edstroem JA, Rubin GM. Sevenless, a cell-specific homeotic gene of drosophila encodes a putative transmembrane receptor with a tyrosine kinase domain. Nature 1987 ; 236 : 55-63.

2. Munnich A, Vaulont S, Marie J. De nouvelles fonctions pour l'AMP cyclique. méde cine/sciences $1985 ; 1$ : 192-7.

3. Zhand R-g, Joachimiak A, Lawson CL, Schevitz RN, Otwinowiski Z, Sigler PB. The crystal structure of trp aporepressor at $1.8 \AA$ shows how binding tryptophan enhances DNA affinity. Nature 1987 ; 327 : 591-6.

$\mathrm{m} / \mathrm{s} n^{\circ} 9 \mathrm{vol}$. 3, novembre 87 
4. Sen R, Baltimore D. Inducibility of $x$ immunoglobulin enhancer-binding protein NF${ }_{x} \mathrm{~B}$ by a post-translational mechanism. Cell $1986 ; 47$ : 921-8.

5. Treisman R. Identification of a protein binding site that mediates transcriptional response of the $c$-fos gene to serum factors. Cell 1986 ; $46: 567-74$

6. Prywes $R, R œ d e r ~ R G$. Inducible binding of a factor to the $c$-fos enhancer. Cell 1986 ; $47: 777-84$

7. Angel $\mathrm{P}$, Imagawa $\mathrm{M}, \mathrm{Chin} \mathrm{R}$, at al. Phorbol ester-inducible genes contain a common cis element recognized by a TPA-modulated transacting factor. Cell 1987 ; 49 : 729-39.

8. Lee W, Mitchell P, Tijian R. Purified transcription factor AP 1 interacts with TPA inducible enhancer elements. Cell $1987 ; 49$ 741-52.

9. Seguin C, Hamer DH. Regulation in vitro of metallothionein gene binding factors. Science 1987 ; 235 : 1383-7.

10. Kingston RE, Schuetz TJ, Larin Z. Heat inducible human factor that binds to a human hsp promoter. Mol Cell Biol $1987 ; 7$ : 1530-4. 11. Sen R, Baltimore D. Multiple nuclear fac tors interact with the immunoglobulin enhancer sequences. Cell 1986 ; 46 : 705-16.

12. Janson L, Bark C, Petterson U. Identification of proteins interacting with the enhancer of human U 2 small nuclear genes. Nucleic Acids Res 1987 ; 15 : 4997-5016.

13. Montminy MR, Bilezikjian LM. Binding of a nuclear protein to the cyclic-AMP response element of the somatostatin gene. Nalure 1987 ; 328 : 175-8.

14. Comb $M$, Birnberg NC, Seasholtz A, Herber E, Goodman HM. A cyclic AMP and phorbol ester-inducible DNA element. Nalure 1986 ; 323 : 353-6.

15. Bodner M, Karin M. A pituitary-specific transacting factor can stimulate transcription from the growth hormone promoter in extracts of non-expressing cells. Cell $1987 ; 50$ : 267-75. 16. Cereghini S, Raymondjean M, Carranca AG, Herbomel P, Yaniv M. Factors involved in control of tissue-specific expression of albumin gene. Cell 1987 ; 50 : 627-38

17. Lenardo $M$, Pierce JN, Baltimore $D$. Protein-binding sites in Ig enhancers determine transcriptional activity and inducibility. Science 1987 ; 236 : 1573-7.

18. Ondek B, Shepard A, Herr W. Discrete elements within the SV-40 enhancer region display different cell-specific enhancer activities. $E M B O J 1987 ; 6$ : 1017-25

19. Rochford R, Campbell BA, Villarreal LP A pancreas specificity results from the combination of polyomavirus and Moloney murine leukemia virus enhancer. Proc Nall Acad Sci USA 1987 ; 84 : 449-53.

20. Schirm S, Jiricny J, Schaffner W. The SV -40 enhancer can be dissected into multiple segments, each with a different cell type specificity. Gene and Development 1987; 1 : 65-74.

21. Distel RJ, Ro HS, Rosen BS, Groves DL, Spiegelman BM. Nucleoprotein complexes that regulate gene expression in adipocyte differentiation : direct participation of $c$-fos. Cell 1987 ; 49 : 835-44.

22. Robertson M. A genetic switch in drosophila morphogenesis. Nalure 1987 ; 327 : 556-7. $\mathrm{m} / \mathrm{s} n^{\circ} 9 \mathrm{vol}$. 3, nooembre 87

\section{BRÈVES}

Il est maintenant possible de dépister avec une très grande sensibilité la " maladie " résiduelle des patients ayant une leucémie ou un lymphome, grâce à la méthode d'amplification d'une séquence spécifique d'ADN par cycles d'extensions d'amorces (en anglais : polymerase chain reaction). A l'aide de deux oligonucléotides de synthèse complémentaires de séquences situées respectivement sur le chromosome 14 et le chromosome 18, de part et d'autre de la zone la plus fréquente de réarrangement dans les translocations $\mathrm{t}(14 ; 18)$, il est possible d'amplifier des centaines de milliers de fois la région hybride $14 ; 18$ située autour de ce point de recombinaison $(\mathrm{m} / \mathrm{s}$ suppl au $n^{\circ}$ 7, vol. 3, p. 12). La translocation $\mathrm{t}(14 ; 18)$ est observée dans $90 \%$ des lymphomes folliculaires humains ; elle réarrange, pense-ton, l'oncogène bcl-2. La méthode d'amplification permet donc, avec une très grande sensibilité, de détecter un très petit nombre de cellules lymphomateuses portant cette translocation, au sein des cellules normales d'un malade en " rémission ", et donc d'évaluer le caractère complet ou partiel de cette rémission. Cette technique est, sans commune mesure, plus sensible que toutes celles, immunologiques, cytogénétiques, ou faisant appel au southern blot classique, utilisées jusqu'alors.

[Lee MS, et al. Nature 1987 ; $237:$ 175-8]

Des métabolites de l'acide arachidonique pourraient être des " seconds messagers" impliqués dans la transmission synaptique. Lorsque l'acide arachidonique est libéré des membranes après activation de la phospholipase $A_{2}$, il est transformé en toute une série de molécules actives qui sont des produits d'oxydation dénommés collectivement les eicosanoïdes. On distingue parmi ces molécules cel- les qui sont produites par l'action de la cyclooxygénase (prostaglandines) de celles produites par l'action des lipooxygénases (leucotriènes, acides hydroxyéicosatétraénoïques ou HETE, lipoxines). Piomelli et al. viennent de montrer que l'action inhibitrice d'un neuromédiateur sur la dépolarisation produite par la 5-hydroxytryptamine au niveau d'un neurone sensoriel du mollusque Aplysie, était due à la libération d'eicosanoïdes du type HETE. La propriété qu'ont ces substances de diffuser librement à travers la membrane cellulaire pourrait en faire des seconds messagers, tout à la fois intra- et intercellulaires, jouant un rôle particulier au niveau des synapses, par exemple dans la potentialisation à long terme de la réponse postsynaptique à un influx nerveux. [Piomelli D, et al. Nature 1987 ; $328: 38-43$ ]

Le gène DMD (de la myopathie de Duchenne) bat vraiment tous les records ! $\mathrm{Sa}$ taille est voisine de $2 \times 10^{6}$ paires de bases (soit $2000 \mathrm{kpb}$, kilo paires de bases), ce qui représente pratiquement le tiers du génome d'Escherichia coli, et près de $1 / 1000$ du génome humain total. La taille moyenne des exons est habituelle $(200 \mathrm{pb}) \ldots$ celle des introns est gigantesque ( $35 \mathrm{kpb}$ !). Le gène est 150 à 200 fois plus grand que le messager; sa transcription in vivo prend probablement... une pleine journée (24 heures !). Au moins $50 \%$ des mutations sont des délétions. Il semble probable que la fréquence des altérations de ce gène est ici en rapport avec son gigantisme ! La " raison " de ce gigantisme est à ce jour obscure. S'il apparaît que cette caractéristique a été conservée au cours de l'évolution, cela indiquera néanmoins qu'elle correspond à un " avantage ", fonctionnel ou génétique, qu'il restera à imaginer ! [Koenig M, et al. Cell 1987; 50 : 509-17] 